\title{
Quantitative Anatomy of Intraorgan Arterial Bed of Kidney According to Segmental Conceptual Model
}

\author{
Zenin O.K. ${ }^{1,}$ Beshulya O.A. ${ }^{2}$ Kafarov E.S. ${ }^{3}$ Vagabov I.U. ${ }^{3}$ Vezirkhanov A.Z. ${ }^{3}$ \\ ${ }^{1}$ Department of Human Anatomy, Penza State University, Penza, Russia \\ ${ }^{2}$ Department of Human Anatomy, And Latin Language, Donetsk National Medical University, Donetsk, Ukraine \\ ${ }^{3}$ Department of Normal and Topographic Anatomy with Surgery, Chechen State University, Grozny, Russia \\ *Corresponding author.Email: zen.olegz@gmail.com
}

\begin{abstract}
The purpose of the study is to determine quantitative indicators of the intraorgan arterial bed of kidney (IABK) of a person, which can be used as a standard morphometric standard in accordance with the segmental conceptual model of arterial bed. IABK of 32 kidneys of people who died from diseases that did not change the kidney angioarchitectonics of 2 age groups: the $2^{\text {nd }}$ period of adulthood - 16, elderly - 16,16 men and 16 women. It was established that IABK is a pseudofractal system, since the values of indicators that quantitatively describe it are associated with many factors (gender, age group, location side, generation number, and division level). Moreover, the morphometric characteristics of the channel are the values of the following indicators: $\mathrm{CM}, \mathrm{FF}, \mathrm{K}$ and $\mathrm{K} 1$. In contrast to the value of the variable FF, the values of the indicators $\mathrm{K}$ and $\mathrm{K} 1$ are not significantly related to gender, age group, side of the body location (left, right), the value of the generation number and the level of division.
\end{abstract}

Keywords: kidney, morphometry, arterial bed, segmental model

\section{INTRODUCTION}

Pathological changes in the structure of the intraorgan arterial bed of the kidney (IABK) are the cause and/or consequence of most kidney diseases [2-6, 9, 19-26]. Therefore, a detailed analysis of the architectonics of IABK could provide invaluable assistance in the early diagnosis of kidney disease. However, at present, there is no unified approach to the analysis of the organization of the arterial bed of the kidney [8, 16]. Information on its main links or chains remains controversial. In addition, today no one needs to be convinced that modern digital methods of intravital imaging of IABK can open up new possibilities for the early diagnosis of its pathology $[1,6$, 17]. However, the lack of a morphometric standard for IABK significantly limits the possibilities of using these methods. [5, 19, 22].

Moreover, it is now becoming apparent that the progress of the medical science will be associated not only with further accumulation of actual knowledge, but also with its creative comprehension. Moreover, it is known that the mathematical theory is the most successful form of knowledge organization, the basis of scientific achievements. Therefore, the development of unifying theory of the structure of the vascular bed, including IABK, and the presentation of all the accumulated material in the form of mathematical models is an urgent task and will require the solution of many problems of practical medicine.

Objective: To determine quantitative indicators of human IABK, which can be used as a morphometric standard, in accordance with the segmental conceptual model of the arterial bed.

\section{METHODS AND MATERIALS}

There were studied the morphometric characteristics of IABK corrosion casts of 32 kidneys of people who died from the diseases that did not change the renal angioarchitectonics (2 age groups: 2nd period of adulthood - 16; elderly - 16 (Materials of the VII AllUnion Conference on Age-Related Morphology, Physiology and Biochemistry of the Academy of Medical Sciences of the USSR, Moscow, 1965), 16 men and 16 women). The corrosion preparations were made according to the standard method [12-16]. A total of 32 corrosive preparations of IABK were manufactured and investigated. The minimum diameter of casts of vascular segments was $0.1 \mathrm{~mm}$ with an accuracy of $0.05 \mathrm{~mm}$. Measurement and registration of arterial segments were carried out in accordance with the widely-known method [11]. Measurement and registration of the bed segments was carried out according to the standard procedure, for the convenience of description, distributing the segments into the proximal (maternal) and distal (daughter) rows. Measurement of the links of the bed was carried out simultaneously, with a gradual step-by-step destruction of the cast. The parameters of each vascular segment were recorded: $\mathrm{D}$ is the diameter of the segment of the proximal row in its central part (in the middle of the distance between the nearest branches) $(\mathrm{mm}), \mathrm{L}$ is the length of the segment of proximal row $(\mathrm{mm})$ - the shortest distance 
between the two branches. To make the description of the channel more formal, it was presented in the form of a connected graph, the vertices of which corresponded to the branch points of the vessels, and the ribs to the vascular segments. The vertices of the graph were numbered arbitrarily and in a manner similar for all investigated cases. The data obtained were entered in special tables containing the following: a) designation of the vertex number, which serves as the beginning of the segment; $b$ ) indication of the number of the vertex that is the end of the segment; c) information on the size of the inner diameter of the segment $(\mathrm{mm})$; d) information about the length of the segment $(\mathrm{mm})$. Data on the structural components of the channel were entered into a computer and used for statistical processing.

IABK was presented in accordance with the segmental conceptual model of the bed as a set of interconnected segments (Fig. 1). To describe such a design, on the basis of the obtained data, the following was determined: $\mathrm{Gr}$, i, $\mathrm{CM}, \mathrm{FF}, \mathrm{K}, \mathrm{K} 1$ : Gr, i.e. generation number, generation linear construction consisting of daughter segments with a larger diameter; $\mathrm{i}$ - level of division - newly formed series of vascular segments; $\mathrm{CM}$ is the coefficient of increase in the number of daughter segments of the distal row, equal to the ratio of the number of segments of the distal row to the number of segments of the proximal row; FF is the form factor - the ratio of the diameter of the vascular segment to its length, $\mathrm{D} / \mathrm{L} ; \mathrm{K}$ is the division coefficient, dmin/D; K1 is the symmetry coefficient, dmax/dmin; dmax is the inner diameter of the daughter segment of the distal row with a large diameter $(\mathrm{mm})$; dmin is the inner diameter of the daughter segment of the distal row with a smaller diameter $(\mathrm{mm})[7,8]$.

The statistical analysis included calculation of the main indicators of the distribution of random variables. To obtain a representative sample, the technique for determining significant median boundaries was used [18]. If the distribution of the values of the studied indicators differed from the normal law, nonparametric criteria were used. The analysis was performed using a licensed package of applied statistical programs, i.e. MedStat, according to the recommendations [18]. The reproduction of the visual image of IABK was carried out using original computer program Vasculograph [10].

\section{RESULTS}

Totally, 9828 arterial segments were measured, comprising 13 generations located at 22 levels of division. The study did not include segments of arteries from which daughter branches do not depart, because there is a high probability of their inaccurate measurement and/or loss of some numerical values. One part of this group, obviously, had a continuation, however, errors in the methodology led to the loss of information about the arterial segments that make up this group. Another part of the arteries could indeed be the final vessels (within the scope of the technique). We called this phenomenon "edge effects". As follows from the foregoing, some of the data was indeed lost during the measurements; therefore, the above approach is logically justified.

The distributions of the obtained values were checked for compliance with the normal distribution law after determining the values of the studied parameters. As a result, it was found that the distribution of the values of all studied indicators (D, dmax, dmin, L, FF, K, and K1) differs from the regular law at the significance level $\mathrm{p}<0.05$. Therefore, for further statistical analysis it is necessary to use nonparametric criteria.

Analyzing the data obtained, it is important to note that the distribution of values of the studied indicators is shifted towards small values. An exception is the distribution of $\mathrm{K}$, which demonstrates the presence of several inhomogeneous groups that make up this generation. The parameter $\mathrm{D}$ characterizes the internal diameters of the segments of the proximal row. Its maximum value is $7.8 \mathrm{~mm}$, the minimum is $0.1 \mathrm{~mm}$. The most common diameter value is $0.5 \mathrm{~mm}$, which differs from the median value of $0.6 \mathrm{~mm}$. The shift of the distribution of diameters towards smaller values is expressed. The parameter dmax describes the inner diameters of large segments of the distal row. Its maximum value is $7.8 \mathrm{~mm}$, the minimum is $0.1 \mathrm{~mm}$. The most common diameter value is $0.1 \mathrm{~mm}$, which differs from the median value of $0.4 \mathrm{~mm}$. The shift of the distribution of diameters towards smaller values is expressed. The parameter dmin characterizes the inner diameters of the smallest segments of the distal row. Its maximum value is $5.4 \mathrm{~mm}$, the minimum is $0.1 \mathrm{~mm}$. The most common diameter value is $0.1 \mathrm{~mm}$, which differs from the median value of $0.3 \mathrm{~mm}$. The shift of the distribution of diameters towards smaller values is expressed. The length of the segments of the proximal row $\mathrm{L}$ varies widely from $26.1 \mathrm{~mm}$ (maximum) to $0.2 \mathrm{~mm}$ (minimum). The most common length is $5 \mathrm{~mm}$, which differs from the median value of $4 \mathrm{~mm}$. As well as for the diameter, this quantity is characterized by a shift in the distribution of values towards smaller values.

The length of the arterial segments $\mathrm{L}$ ranged from $47 \mathrm{~mm}$ (maximum) to $0.3 \mathrm{~mm}$ (minimum). The most common length is $3 \mathrm{~mm}$, which differs from the average value of $5.6 \mathrm{~mm}$. As well as for the diameter, this quantity is characterized by a shift in the distribution of values towards smaller values. A large scatter in the values of the inner diameter and length of segments determines the pronounced variability of the form factor $\mathrm{FF}(\mathrm{M} \pm \mathrm{m})$. Its maximum value of 9.5 is the minimum -0 . The value of $\mathrm{K}$ ranges from 7 (maximum value) to 0 (minimum value). The most common value is 0.5 , which does not coincide with the value of 0.5 median. The distribution of indicators is biased towards average values. The value of $\mathrm{K} 1$ ranges from 12 (maximum value) to 0 (minimum value). The most common value is 1 , which almost coincides with the value of 1.2 medians. The distribution of indicators is biased towards lower values. The analysis of the values of the $\mathrm{CM}$ indicator, i.e. the coefficient of increase in the number of daughter segments of the distal row, indicates the presence of an overwhelming number of dichotomies $98 \pm 0.14 \%$ (9637 items), trichotomy - $1.91 \pm 0.14 \%$ 
(180 items), quadratomy $-0,09 \pm 14 \%$ (9 items) and even pentatomies - $0.01 \pm 0.14 \%$ (1). The presence of pentatomies can be considered as an artifact.

To establish correlations between the studied parameters, nonparametric analysis was used. Moderate severity (statistically significantly different from 0 ) correlation relationships were established between: positive i-Gr, LD, L-dmax, L-dmin, K-dmax, K-dmin, K1dmax, FF-D, FF-dmax, FF-dmin; negative D-Gr, D-i, dmax-Gr, dmax-i, dmin-Gr, dmin-i, FF-Gr, FF-i, FF-L. Strong links are established between positive dmax-D, dmin-D, dmin-dmax.

The analysis of location of the segments by generation numbers and division levels showed that the equation determining the relationship between the absolute number of segments and the division level make it possible to reliably describe this relationship up to the 4th generation number and 9th division level, then the "edge effects" introduce a large error. Taking into account the above relationships of the $\mathrm{CM}$ value with the generation number and the division level, we analyzed the percentage ratio of branches with different $\mathrm{CM}$ values depending on the generation number and the division level.

The following interesting data were obtained: branches with a large CM value (i.e., trichotomy, quadrithomy and pentatomy) belong to the initial generations and are located at more proximal fission levels. The results of correlation analysis confirm that the values of indicators included in the composition of trichotomies, quadrithomies and pentatomies, such as: D, dmax, dmin, L, FF and K1 are greater, and the values of $\mathrm{K}$ are less than those of dichotomies $(\mathrm{CM}=2)$. The diameters of the arterial segments of the VARP decrease with an increase in the division level and generation number, but the values of the $\mathrm{K}$ index increase. The larger the diameter of the proximal segment, the higher the diameters of the larger and smaller segments of distal row, and, accordingly, the larger the diameter of larger segment of the distal row, the larger the diameter of the smaller one. The same statement is true for the length of the segments: the longer the length of the segment of the proximal row, the greater it is for the larger and smaller segments of the distal row. The expressed negative connections between $\mathrm{D}$, dmax and dmin are explained by the formulas for calculating the last indicator, therefore, it can be argued that with an increase in the D indicators, the morphological component of the hemodynamic resistance of a segment of a given diameter decreases.

As a result of the regression analysis of the dmax-D, dmin-D, L-D bonds, the following equations were established: $\mathrm{dmax}=0.81 \mathrm{D}-0.08, \mathrm{R} 2=0.87 ; \mathrm{dmin}=$ $0.62 \mathrm{D}-0.06, \mathrm{R} 2=0.78$. The dmax-D and dmin-D bonds are accurately described by linear equations. To describe the L-D connection, a fourth degree polynomial equation was chosen, because, in comparison with others, it most accurately describes this connection, and the fourth degree because, as follows from the previously given data: $\mathrm{L}=0.014 \mathrm{D} 4+0.14 \mathrm{D} 3-0.6 \mathrm{D} 2+3.0 \mathrm{D}+2.56, \mathrm{R} 2=0.2496$, the accuracy of the measurements obtained can be fully guaranteed up to the fourth generation number. It is possible that the studied set of values of the studied indicators consists of several independent samples, and by isolating these samples, it would be possible to increase the accuracy of the regression equations.

Therefore, the hypothesis about the belonging of independent samples to one general population was further tested. The criteria used were the Mann-Whitney U Test and Kruskal-Wallis ANOVA Test. Independent samples of the values Gr, i, CM, D, dmax, dmin, L, FF, K, and K1 were analyzed for their possible relationship with gender, age group, and side of the kidney.

Totally, 4745 arterial segments of men and 5083 segments of women were studied, as well as 4763 segments belonging to IABK persons of the 2nd period of adulthood and 3237 of the elderly group. The value of $\mathrm{FF}$ indicator is greater in men $-0.2 \pm 0.003$, than in women $-0.16 \pm 0.006$ ( $\mathrm{p}<0.001)$, respectively. The values of $\mathrm{Gr}$ and $\mathrm{L}$, on the contrary, are greater in women $5 \pm 0.03$ and $4.6 \pm 0.05$ than in men $-4 \pm 0.03(\mathrm{p}=0.04)$ and $3.4 \pm 0.05(\mathrm{p}<0.001)$, respectively. The data presented indicate that the linear sizes of IABK in women are on average larger than in men. The central trends in the values of the indicators $\mathrm{D}, \mathrm{dmax}$ and dmin differ at the significance level $\mathrm{p} \leq 0.05$, which is confirmed by the differences in distribution of the values of these indicators in men and women. The number of segments having a diameter with the value in the range from 0 to $1 \mathrm{~mm}$ is relatively larger in women than in men. The shapes of the curves that describe the nature of the decrease in the diameters of vascular segments with an increase in the generation number and division level also differ in men and women. The values of the remaining studied indicators (i, CM, K and K1) do not significantly differ among representatives of different sexes.

The values of IABK indicators are as follows: $\mathrm{Gr}, \mathrm{i}, \mathrm{K}$ and FF are less in persons of the 2 nd period of adulthood $4 \pm 0.03 ; 9 \pm 0.05 ; 0.5 \pm 0.004$ and $0.16 \pm 0.003$ than in the elderly $-5 \pm 0.03(\mathrm{p}<0.001) ; 9.1 \pm 0.07(\mathrm{p}=0.01) ; 0.51 \pm 0.006$ $(\mathrm{p}<0.001)$ and $0.2 \pm 0.02(\mathrm{p}<0.001)$, respectively. Whereas the values of the variables are the following: $D$, dmax, dmin and $\mathrm{K} 1$ in individuals of the 2 nd period of adulthood are greater than in elderly persons. The values of $\mathrm{CM}$ parameter do not significantly differ $\mathrm{p}=0.06$.

Thus, it can be argued that the linear dimensions of the arterial segment that make up the IABK decrease with age. In addition, based on the above facts, it can be assumed that people with large linear dimensions of the arterial segments of the arteries, its components, are more likely to survive to the old age.

The following differences in the central tendencies of the values of indicators are identified: D, dmax and dmin between the segments of the left and right IABK $(\mathrm{p} \leq 0.05)$ are established, which is confirmed by the differences in the distributions of the values of these indicators between the left and right segments. The shape of the curves describing the nature of the decrease in the values of diameters of the vascular segments with an increase in the generation number and the level of division also differ in the left and right IABK. It was found that the values of the variables $\mathrm{K} 1$ and $\mathrm{FF}$ of the arterial segments belonging to 
the left IABK are significantly larger than the right $\mathrm{p}=0.02$ and $\mathrm{p}<0.001$, respectively. And vice versa, the values of the variable $\mathrm{CM}$ of the right segments are significantly larger than the left ones $\mathrm{p}<0.001$.

Next, the hypothesis of equality of the average independent samples was tested. The Kruskal-Wallis ANOVA test was used. It was found out that the largest number of branches constituting IABK is accounted for by dichotomies $-98 \%, \mathrm{CM}=3$ make up $1.91 \%$, and $\mathrm{CM}=4$ constitute $0.09 \%$. During the study, 2 branches with $\mathrm{CM}=5$ were found, which can be considered as an artifact. The analysis of the results confirms the statement that branches with $\mathrm{CM}=3$ and $\mathrm{CM}=4$ are located at proximal levels of division. The value of FF does not significantly differ in arterial segments belonging to branches with different $\mathrm{CM}$ values. It was found that the arterial segments that make up the dichotomy are characterized by the minimum values of internal diameters, lengths and $\mathrm{K} 1$, the maximum - K. The following characteristics are typical for trichotomies: medium - diameters, lengths, $\mathrm{K}$ and $\mathrm{K} 1$. For branches with $\mathrm{CM}=4$ - minimum values: $\mathrm{K}$, maximum - diameters, lengths and $\mathrm{K} 1$.

The results of the analysis can be further considered as a standard for the mathematical modeling of the vascular bed.

\section{CONCLUSION}

Thus, the traditional descriptive anatomy of IABK does not make it possible to establish the morphometric standard of the IABK norm, since it is practically impossible to identify various links of the bed on a corrosive preparation, and also because it is better to use relative indices as reference values than the absolute ones. It is more expedient to use the values of relative indicators, which quantitatively describe the conceptual models of IABK.

Human IABK is a pseudofractal system, since the values of indicators that quantitatively characterize it are associated with many factors (gender, age group, location, generation number and division level). Moreover, the morphometric characteristics of the channel are the values of the indicators $\mathrm{CM}, \mathrm{FF}, \mathrm{K}$ and $\mathrm{K} 1$. In contrast to the value of the variable $F F$, the values of the indicators $K$ and $\mathrm{K} 1$ are not significantly related to gender, age group, side of the body location (left, right), the value of the generation number and the level of division.

As a structural unit (fractal) of IABK, one can consider arterial branching, which consists of the maternal arterial segment, several daughter branches and, in fact, the branch item, i.e. quadritomy, trichotomy and dichotomy. It was found that the quadrithomies accounted for $0.09 \pm 14 \%$ of the total number of branches, trichotomy was $1.91 \pm 0.14 \%$ and dichotomies was $98 \pm 0.14 \%$ (9637 items). Therefore, it is logical to imagine dichotomy as the main structural unit (fractal) of IABK.

\section{ACKNOWLEDGMENT}

The article was published in the framework of RFBR Grant according to the Agreement No.18-29-09118.

\section{REFERENCES}

[1] F.R. Asfandiyarov, E.S. Kafarov, A.V. Stabred, Topographic anatomy of renal artery, vein and pelvis, Bull. of new med. technol. XV111(2) (2011) 40-41.

[2] I.U. Vagabov, E.S. Kafarov, Variant anatomy of renal artery according to multispiral computed tomography, Morphol. 3 (2018) 52.

[3] I.U. Vagabov, E.S. Kafarov, Types of branching of the intraorgan arterial vessels of human kidney, J. Morphol. 3 (2018) 131.

[4] I.U. Vagabov, E.S. Kafarov, Spatial and tier organization of human kidney arterial system, Journal Collection of materials of International Scientific Conference dedicated to R.I. Asfandiyarova, 2018, pp. 24-28.

[5] I.V. Vilkhova, Morphological and topographic features of archeology of human beings (X-ray and ultrasound studies), Ph.D. Thesis, 2002, 19 p.

[6] I.V. Gaivoronsky, G.I. Nichiporuk, Clinical anatomy of blood vessels and nerves, ELBI-SPb, St. Petersburg, 2007, $144 \mathrm{p}$.

[7] A.V. Dmitriev, O.K. Zenin, Yu.V. Dovgyallo, Conceptual models of tree-like arterial bed, Bull. of Emergency and Restorative Med. 8(2) (2007) 297-302.

[8] O.K. Zenin Human arterial system in numbers and formulas, Donbass, Donetsk, 2002, 196 p.

[9] O.K. Zenin, E.L. Kryukov, D.V. Remizov, Computer program - Vasculograpx.exe, which simulates the intraorgan channels of functionally different internal organs of a person, Applied Aspects of Morphology. Topical Aspects of Functional Morphology and Integrative Anthropology, Materials of Science and Practice. conf., Vinnitsya, 2009, pp. 112-114.

[10] O.K. Zenin, Morphological and functional principles of organization of arterial bed of great circle of blood circulation, Ph.D. Thesis, Kiev, 2005, 468 p.

[11] E.S. Kafarov, S.V. Fedorov, I.U. Lechiev, Comparative characteristics of venous vessels of human kidneys according to x-ray angiography and computed tomography studies, Morphol. 152(4) (2017) 32-37.

[12] E.S. Kafarov, I.U. Vagabov, S.-M. N. Mezhidov et al., Structural changes in the walls of the renal veins during aging, Materials of the conference of the faculty devoted to the 80th anniversary of Chechen State University, 2018, pp. 150-154. 
[13] E.S. Kafarov, L.A. Udochkina, L.R. Elzhurkaeva, Topographic and anatomical analysis of the tubular structures of kidney gates according to the data of corrosion preparations, J. of the Herald of the Med. Inst. 1(13) (2018) 26-30.

[14] E.S. Kafarov, Structural transformations of venous vessels of human kidney in adulthood, the elderly and senile ages, Ph.D. Thesis, Ufa, 2014, 341 p.

[15] E.S. Kafarov, F.R. Asfandiyarov, M.N. Trizno, Types of branching of arterial and venous vessels of the kidney, Morphol. Bull. 3-4 (2008) 41-42.

[16] O.O. Mochalov, Variability of architectonics of blood vessels of kidney, Ph.D. Thesis, 2006, 164 p.

[17] Yu.E. Lyakh, V.G. Guryanov, V.N. Khomenko, O.A. Panchenko, Fundamentals of computer biostatistics: analysis of information in biology, medicine and pharmacy with the MedStat statistical package, D.: Papakitsa E.K., 2006, 214 p.

[18] A. Marcin, W. Andrzej, The Management of Atherosclerotic Renovascular Disease, Kidney Blood 34 (2011) 277-283.

[19] R.E. Batt, J. Yeh., J. Minim, Novel surgical approach to retroperitoneal surgery by Zacharopoulou, Batt, Invasive, Gynecol. 16(5) (2009) 659.

[20] A.E. Burchell, J. Rodrigues, M. Charalambos et al., Comprehensive first-line magnetic resonance imaging in hypertension: experience from a single- center tertiary referral clinic, J. of Clin. Hypertension 19(1) (2017) 13-22.

[21] G.G. Callender, Posterior retroperitoneoscopic adrenalectomy, Adv. Surg 43 (2009) 147-157.

[22] E.S. Kafarov, L.A. Udochkina K.M. Bataev, S.V. Fedorov, Options for the Formation of Renal Human Veins, Int. J. of Engineer. and Advan. Technol. 8(4) (April 2019). ISSN: 2249-8958.

[23] T.L. Kline, M.E. Edwards, P. Korfiatis et al., Semiautomated segmentation of polycystic kidneys in T2-weighted MR images, AJR 7 (2016) 605-613.

[24] S.J. Mann, T.A. Sos, Misleading results of randomized trials: the example of renal artery stenting, J. Clin. Hypertens 12 (2010) 1-2.

[25] G.C. Kane, N. Xu, E. Mistrik, Renal artery revascularization improves heart failure control in patients with atherosclerotic renal artery stenosis, Nephrol. Dial. Transplant. 25 (2010) 813-820.

[26] B.A. Urban, Three-dimensional volume-rendered CT angiography of the renal arteries and veins: normal anatomy, variants and clinical applications, vol. 21, 2006, pp. 373-382. 